\title{
COMMUTATIVE ABSOLUTE SUBRETRACTS
}

\author{
E. JESPERS and M. M. PARMENTER
}

(Received 13 May 1991)

Communicated by P. Schultz

\begin{abstract}
Directly indecomposable absolute subretracts that are commutative Noetherian rings are described. This is an application of our main result characterizing unital directly indecomposable absolute subretracts which contain a maximal ideal with nonzero annihilator.
\end{abstract}

1991 Mathematics Subject Classification (Amer. Math. Soc.): Primary 16 D 50.

Throughout this paper, all rings are associative and commutative. The variety generated by a ring $R$ is denoted $\operatorname{Var}(R)$ (cf. [7]).

Recently, several authors $[2,4,5]$ have studied the notion of absolute subretract. Recall that a ring $R$ is said to be an absolute subretract if for every ring $S$ in $\operatorname{Var}(R)$ and every ring monomorphism $f: R \rightarrow S$, there exists a ring morphism $g: S \rightarrow R$ such that $g f$ is the identity mapping on $R$. In [4], Gardner and Stewart characterized directly indecomposable absolute subretracts $R$ with $R^{2}=\{0\}$, and gave an example of a special principal ideal ring which is an absolute subretract. Then Jespers [5] obtained necessary and sufficient conditions for a finite special principal ideal ring (of characteristic different from $2^{n}$ ) to be an absolute subretract, also obtaining results for the infinite and characteristic $2^{n}$ cases.

We first show that in a unital directly indecomposable absolute subretract $R$, the set of zero divisors is a maximal ideal $M$ and $R / M$ is finite. If, moreover, $M$ has nonzero annihilator (denoted $\operatorname{Ann}(M)$ ), we obtain necessary and sufficient conditions for $R$ to be an absolute subretract. As an immediate consequence, a characterization of Noetherian directly indecomposable 
absolute subretracts is obtained. The latter is then applied to Noetherian contracted monoid algebras.

LemMa $1[2,4]$. Let $R$ be a directly indecomposable absolute subretract. If $I$ and $J$ are nonzero ideals of $R$, then $I \cap J \neq\{0\}$.

Proposition 2. Let $R$ be a unital ring. If $R$ is a directly indecomposable absolute subretract, then the set of zero-divisors of $R$ is a maximal ideal $M$ and $R / M$ is finite.

Proof. Let $M$ be the set of zero divisors. Because of Lemma 1, one easily verifies that $M$ is an ideal of $R$. Clearly $R / M$ is a domain. If $R / M$ is finite, then $R / M$ is a field and thus $M$ is a maximal ideal. In the remainder, we show that $R / M$ has to be finite.

Suppose the contrary. Then $R / M$, being an infinite domain, satisfies only identities $f(X)=0$ where $f(X) \in p Z[X], p=\operatorname{char}(R / M)$. If $p=0$, we have shown that $R / M$, and hence $R$, satisfies no nontrivial polynomial identities. If $p \neq 0$, any nontrivial polynomial identity satisfied by $R$ must be of the form $0=p^{n} f(X) \in \mathbf{Z}[X], n \geq 1$, where $f(X) \notin p \mathbf{Z}[X]$. We claim that in this case $\operatorname{char}(R) \mid p^{n}$. If not, then $p^{n} \neq 0$ in $R$ so $f(X)$ is in $M$ for all choices of $X$ and $f(X)=0$ in $R / M$. But we saw before that this implies $f(X) \in p \mathbf{Z}[X]$, a contradiction.

In either case (cf. [6]), $\operatorname{Var}(R)$ contains all central extensions of $R$. As in [8] and [4], we focus our attention on the localization of the polynomial ring in one variable $R[x]$ obtained by inverting all monic polynomials, and denote this by $T$. Since $R$ is an absolute subretract, there is a homomorphism $g: T \rightarrow R$ extending the identity map on $R$. Say $g(x)=r$. Then, $g(x-$ $r)=0$, a contradiction since $x-r$ is invertible. This finishes the proof.

CoRollary 3. Let $R$ be a unital directly indecomposable absolute subretract and let $M$ be the ideal of zero divisors. If $\operatorname{Ann}(M) \neq 0$, then $\operatorname{Ann}(M)$ is the minimum nonzero ideal of $R$.

Proof. Say $\operatorname{Ann}(M) \nsubseteq I$ for some nonzero ideal $I$ of $R$. Choose $0 \neq$ $m \in \operatorname{Ann}(M)$ with $m \notin I$. By Lemma 1 , there exists $r \in R$ such that $0 \neq$ $r m \in I$. Proposition 2 then says that $r^{n-1}=1+\alpha$ for some $\alpha \in M$ where $n=|R / M|$. But this implies that $m=r^{n-1} m \in I$, a contradiction.

Lemma 4. Let $R$ be a unital directly indecomposable absolute subretract with $M$ the ideal of zero divisors. Assume $M \neq\{0\}$ and $\operatorname{Ann}(M) \neq\{0\}$. If $|R / M|>2$, then $M=\operatorname{Ann}(M)$, that is $M^{2}=\{0\}$. 
Proof. Since $R / M$ has more than two elements, there exist $u, u^{\prime} \notin M$ with $u-u^{\prime} \notin M$. Also, by Corollary $3, \operatorname{Ann}(M)=R x$ for some $0 \neq x \in R$.

Let $S=\{(a, a+j) \in R \times R \mid j \in M\}$ and let $I$ be the principal ideal generated by $\left(u x, u^{\prime} x\right)$ in $S$. Note that, since $M x=0, I=$ $\left\{\left(a u x, a u^{\prime} x\right) \mid a \notin M\right\} \cup\{0\}$, and that $T=S / I$ is in $\operatorname{Var}(R)$. Define $f: R \rightarrow T$ by $f(r)=(r, r)+I$. Clearly $f$ is a homomorphism. Suppose $f(r)=0$ for some $r \neq 0$, that is $(r, r) \in I$. Then $0 \neq r=a u x=a u^{\prime} x$ for some $a \notin M$, so $a\left(u-u^{\prime}\right) x=0$. Since $a\left(u-u^{\prime}\right) \notin M$, that is, $a\left(u-u^{\prime}\right)$ is not a zero divisor, it follows that $x=0$, a contradiction. Thus $f$ is a monomorphism.

We now show that every principal ideal $T t$ of $T$ intersects $f(R)$ nontrivially, where $0 \neq t=(a, b)+I$.

First consider the case where $a, b \in R x$, that is, $a=v x, b=w x$ and either $v$ or $w$ is not in $M$. Let $s=\left(u^{\prime}-u\right)^{n-2}(v-w)$ where $n=|R / M|$. Note that $s\left(u^{\prime}-u\right) x=\left(u^{\prime}-u\right)^{n-1}(v-w) x=(1+\alpha)(v-w) x$ for some $\alpha \in M$. Hence

$$
s\left(u^{\prime}-u\right) x=(v-w) x .
$$

Therefore $(v+s u) x=\left(w+s u^{\prime}\right) x$, and so $0 \neq(a, b)+I=(v x, w x)+$ $s\left(u x, u^{\prime} x\right)+I=\left((v+s u) x,\left(w+s u^{\prime}\right) x\right)+I \in f(R)$.

Next assume that either $a \notin R x$ or $b \notin R x$, for example, say, $a \notin R x$. Because $M a \neq 0$ and $R x$ is minimum, there exists $r \in M$ with $r a=x$. Hence $(r, 0) \in S$ and $(r, 0)(a, b)+I=(x, 0)+I$ belongs to $T t$. Note that $(x, 0) \notin I$ since $(x, 0)=\left(a u x, a u^{\prime} x\right)$ implies $a u^{\prime} x=0$. As $u^{\prime}$ is not a zero divisor, this yields $a x=0$ and thus $x=a u x=0$, a contradiction. By the previous case, we know that $(x, 0)+I$ belongs to $f(R)$.

Since $R$ is an absolute subretract, it follows that $f(R)=T$. Hence, for every $m \in M,\left(u m+x, u^{\prime} m+x\right)+I \in f(R)$. Thus $\left(u m+x, u^{\prime} m+x\right)=$ $(r, r)+\left(a u x, a u^{\prime} x\right)$ for some $r \in R$. It follows that $u m-u^{\prime} m \in \operatorname{Ann}(M)$. Since $u-u^{\prime}$ is not a zero divisor, we conclude that $m \in \operatorname{Ann}(M)$, so $M \subseteq$ $\operatorname{Ann}(M)$. Since $M \neq 0, \operatorname{Ann}(M) \subseteq M$, and the result follows.

We next show that the characteristic 2 case can be settled in the same way.

LeMma 5. Let $R$ be a unital directly indecomposable absolute subretract with $M$ the ideal of zero divisors. Assume $M \neq\{0\}$ and $\operatorname{Ann}(M) \neq\{0\}$. If $|R / M|=2$, then $M=\operatorname{Ann}(M)$.

Proof. Let $S=\{(a, b, c) \mid a, b, c \in R, a-b$ and $b-c \in M\}$. Note that $S \in \operatorname{Var}(R)$. Let $\operatorname{Ann}(M)=R x, x \neq 0$, and define

$$
I=\{(0,0,0),(0, x, x),(x, 0, x),(x, x, 0)\} .
$$

Observe that $I$ is an ideal of $S$ and let $T=S / I$. 
Define $f: R \rightarrow T$ by $f(r)=(r, r, r)+I$. Clearly $f$ is a monomorphism. We claim that every nonzero principal ideal of $T$ intersects $f(R)$ nontrivially. For this, let $0 \neq t=(a, b, c)+I$. If $a, b, c$ are all in $R x=\{0, x\}$, then since $t \neq 0$, we have $t=(x, x, x)+I=f(x)$. So assume, for example, that $a \notin R x$. Then $M a \neq 0$ and thus $M a \supseteq R x$ since $R x$ is minimum. So $r a=x$ for some $r \in M$. Hence $(r, 0,0) \in M$ and $(r, 0,0)(a, b, c)+I=(x, 0,0)+I=(x, x, x)+I=f(x)$ is in $T t$, and the claim is proved. Since $R$ is an absolute subretract, we conclude that $f(R)=T$. Hence for every $m \in M,(m, m, 0)+I \in f(R)$. Consequently, $m \in R x$ so $M \subseteq \operatorname{Ann}(M)$. Since $M \neq 0, \operatorname{Ann}(M) \subseteq M$ and the result follows.

We are now ready to prove our main result.

Theorem 6. Let $R$ be a unital ring with maximal ideal $M$ such that $\operatorname{Ann}(M) \neq\{0\}$. Then $R$ is a directly indecomposable absolute subretract if and only if $R$ is finite and $M=R x, x^{2}=0$, for some $x$ in $R$.

Proof. First we show that the conditions are sufficient. If $M \neq\{0\}$, this follows from Proposition 2 in [5]. The case $M=\{0\}$, that is $R$ is a finite field, is proved in the same way, but we will sketch it here for completeness. So let $R$ be a finite field and say $f: R \rightarrow T$ is a monomorphism where $T \in \operatorname{Var}(R)$. Although $T$ itself may not have a multiplicative identity, it has a direct summand $T_{1}$ such that $f(R) \subseteq T_{1}$ and $T_{1}$ shares the same multiplicative identity as $f(R)$. Choose $N$ an ideal of $T_{1}$ maximal such that $f(R) \cap N=\{0\}$. Then $T_{1} / N$ is a field satisfying the same polynomial identities as $R$ and $R$ is embedded in $T_{1} / N$. Hence $R \simeq T_{1} / N$ and the result follows.

To prove the necessity of the conditions, note that because of Proposition 2 and the assumptions, $M$ is the set of zero divisors of $R$ and $R / M$ is a finite field. The result then follows from Lemmas 4 and 5 .

Corollary 7. Let $R$ be a unital directly indecomposable ring. Then $R$ is a Noetherian absolute subretract if and only if $R$ is a finite field or $R$ is a finite local ring with maximum ideal $M=R x$ and $x^{2}=0$ for some $0 \neq x \in R$.

Proof. Assume $R$ is a Noetherian absolute subretract. Then by the assumptions and Proposition 2, the set of zero divisors $M$ is a finitely generated maximal ideal. Hence by Lemma $1, \operatorname{Ann}(M) \neq\{0\}$. The result now follows from Theorem 6 .

We conclude with an application to contracted monoid algebras. For terminology and notation we refer to [1]. 
COROLLARY 8. Let $k$ be a field and $S$ a commutative monoid with identity element $e$ and zero element $\theta \neq e$. Then the contracted monoid algebra is a Noetherian directly indecomposable absolute subretract if and only if $k$ is a finite field and one of the following conditions is satisfied.

(i) $S=\{e, s, \theta\}, s^{2}=\theta, s \neq \theta, s \neq e$. In particular, $k_{0}[S]=$ $k[x] /\left(x^{2}\right)$.

(ii) $\operatorname{char}(k)=2, S=\{e, s, \theta\}, s^{2}=e$. In particular, $k_{0}[S] \cong k\left[Z_{2}\right], a$ group algebra of the cyclic group of order 2.

(iii) $S=\{e, \theta\}$. In particular, $k_{0}[S] \cong k$.

Proof. First note that $k_{0}[S]$ is a field if and only if $S=\{e, \theta]$, which is precisely case (iii). Because of Corollary 7 and the results in [3], the proof now goes exactly as the proof of Corollary 4 in [5].

\section{References}

[1] A. H. Clifford and G. B. Preston, The Algebraic Theory of Semigroups, vol. I (Amer. Math. Soc., Providence, RI, 1961).

[2] B. A. Davey and L. G. Kovács, "Absolute subretracts and weak injectives in congruence modular varieties', Trans. Amer. Math. Soc. 297 (1986), 181-196.

[3] F. Decruyenaere, E. Jespers and P. Wauters, 'On commutative principal ideal semigroup rings', Semigroup Forum 43 (1991), 367-377.

[4] B. J. Gardner and P. N. Stewart, 'Injective and weakly injective rings', Canad. Math. Bull. 31 (1988), 487-494.

[5] E. Jespers, 'Special principal ideal rings and absolute subretracts', Canad. Math. Bull. 34 (1991), 364-367.

[6] R. N. McKenzie, G. F. McNulty and W. F. Taylor, Algebras, Lattices, Varieties, vol. I (Wadsworth, Belmont, CA, 1987).

[7] C. Procesi, Rings with polynomial identities (Marcel Dekker, New York, 1973).

[8] R. Raphael, 'Injective rings', Comm. Algebra I (1974), 403-414.

\section{Department of Mathematics and Statistics \\ Memorial University of Newfoundland}

St. John's, Newfoundland

Canada A1C 5S7 